\title{
Association between four common microRNA polymorphisms and the risk of hepatocellular carcinoma and $\mathrm{HBV}$ infection
}

\author{
JIAN-TAO KOU, HUA FAN, DONGDONG HAN, LIXIN LI, PING LI, \\ JIQIAO ZHU, JUN MA, ZHI-HUA ZHANG and QIANG HE
}

Department of Hepatobiliary Surgery, Beijing Chaoyang Hospital, Capital Medical University, Beijing 100020, P.R. China

Received September 4, 2013; Accepted April 10, 2014

DOI: $10.3892 / \mathrm{ol} .2014 .2257$

\begin{abstract}
RNAs (miR/miRNAs) have been demonstrated to function as tumor suppressors and oncogenes, and miRNA polymorphisms may have a role in cancer development. The present study aimed to investigate the association between the miR-146aG >C, miR-149C >T, miR-196a2C >T and miR-499A > G polymorphisms and the risk of hepatocellular carcinoma (HCC) and hepatitis B virus (HBV) infection. A total of 271 patients with HCC and 532 healthy control participants were enrolled in the present study. miR-146aG $>C$, miR-149C $>$ T, miR-196a2C $>$ T and miR-499A $>$ G polymorphisms were genotyped using the polymerase chain reaction-restriction fragment length polymorphism method. A significant difference was identified in the genotype frequency of miR-196a2C $>\mathrm{T}$ in the patients in the case group compared with the control group $\left(\chi^{2}=6.88 ; \mathrm{P}=0.032\right)$. Compared with the CC genotype, the miR-196a2 TT genotype was associated with a significantly reduced risk of HCC [odds ratio (OR), 0.62; 95\% confidence interval (CI), 0.38-0.99], and a significantly reduced risk was also found in the dominant (OR, 0.69; 95\% CI, 0.49-0.98) and recessive (OR, 0.70; 95\% CI, 0.46-1.02) models. Moreover, individuals with HBV who were carrying the miR-196a2 CT and TT genotypes had a significantly reduced risk of HCC (OR, 0.62; 95\% CI, 0.41-0.95; and OR, 0.39; 95\% CI, 0.20-0.73, respectively). In conclusion, the present study found that the miR-196a2C $>$ T polymorphism has a protective effect in patients with HCC, particularly in those with HBV infection.
\end{abstract}

\section{Introduction}

Hepatocellular carcinoma (HCC) is the fifth most common type of malignant tumor worldwide and has the second highest

Correspondence to: Professor Qiang He, Department of Hepatobiliary Surgery, Beijing Chaoyang Hospital, Capital Medical University, 8 Gongren Tiyuchang Nanlu, Chaoyang, Beijing 100020, P.R. China

E-mail: heqiang_cmu@163.com

Key words: microRNA, polymorphisms, hepatocellular carcinoma, hepatitis B virus mortality rate, with an estimated 293,318 new cases in China per year (1). The primary risk factors for HCC are chronic hepatitis $\mathrm{B}$ virus (HBV) and hepatitis $\mathrm{C}$ virus (HCV) infection; however, only $\sim 10 \%$ of patients infected with $\mathrm{HBV}$ and HCV develop HCC during their lifetime $(2,3)$. Thus, certain genetic and environmental factors may also be involved in the development of HCC (2).

microRNAs (miR/miRNAs) are a novel class of endogenous, non-coding RNAs that regulate gene expression at the post-transcriptional level through repressing translation or decreasing mRNA stability $(4,5)$. miRNAs have been demonstrated to play a role in several processes, including development, apoptosis, proliferation and differentiation, in the eukaryotic cells of various organisms $(6,7)$. A number of previous studies have analyzed the association between miRNAs and the susceptibility and prognosis of various types of human cancer (8-10). The miR-146aG $>C$, miR-149C $>\mathrm{T}$, miR-196a $2 \mathrm{C}>\mathrm{T}$ and miR-499A $>\mathrm{G}$ polymorphisms have been reported to be associated with various types of cancer, including lung, breast, colorectal and gastric cancer (11-14). Several more recent studies have reported that miRNA polymorphisms are associated with the risk of HCC; however, the results are inconsistent (15-18). Moreover, several studies have demonstrated that miRNAs act as repressors in viral infection pathways, and that viruses have miRNAs which regulate gene expression, thus miRNAs contribute to the pathogenicity of the virus $(20,21)$. miRNAs may therefore be key regulators in host-virus interactions and in the regulation of viral replication. In the present study, it was hypothesized that polymorphisms in miR-146aG $>C$, miR-149C $>$ T, miR-196a $2 \mathrm{C}>\mathrm{T}$ and miR-499A $>$ G may have an effect on the risk of HCC and the interaction with HBV infection. In order to investigate this hypothesis, a case-control study was performed to investigate the association between four common miRNA polymorphisms and the risk of HCC.

\section{Materials and methods}

Study population. A total of 302 individuals were periodically enrolled in the present study between January 2010 and February 2012. HCC diagnoses were based on liver biopsies or at least two radiological tests for HCC, including abdominal ultrasound, spiral computed tomography, magnetic resonance imaging and hepatic angiography, or by increased $\alpha$-fetoprotein 
Table I. Primer sequences used for miRNA amplification.

\begin{tabular}{llc}
\hline Gene variants & \multicolumn{1}{c}{ Primer sequence (5'-3') } & Product, bp \\
\hline miR-146aG $>C$ & & 147 \\
Forward & & \\
Reverse & 5'-CAA AGT CTT CACTTC CCT GCC A-3' & 263 \\
miR-149C $>\mathrm{T}$ & 5'-GAT GTT TAA CTC CTC TCC ACG TGA TC-3' & \\
Forward & & 149 \\
Reverse & 5'-CTG GCT CCG TGT CTT CAC TC-3' & \\
miR-196a2C $>$ T & 5'-TGA GGC CCG AAACAC CCG TA-3' & \\
Forward & & 146 \\
Reverse & 5'-CCC CTT CCC TTC TCC TCC AGA TA-3' \\
miR-499A $>$ G & 5'-CGA AAA CCG ACT GAT GTA ACT CCG-3' \\
Forward & & \\
Reverse & 5'-CAA AGT CTT CAC TTC CCT GCC A-3' & \\
\hline
\end{tabular}

miR/miRNA, microRNA.

levels ( $\geq 200 \mu \mathrm{g} / \mathrm{ml}$ ). The control group consisted of 568 individuals randomly selected from the health examination center at Beijing Chaoyang Hospital of Capital Medical University (Beijing, China). None of the control subjects had a history of cancer, liver disease, kidney disease, coronary artery disease or other metabolic disorders.

Serum hepatitis B surface antigen and anti-HCV antibody levels were assessed with a microparticle enzyme immunoassay using commercial assay kits to determine the infection of HBV or HCV. The clinical characteristics of the patients with HCC were obtained using medical records. The demographic characteristics were collected using a self-designed questionnaire, which included questions on smoking status and alcohol consumption. The present study was approved by the Medical Ethical Committee of Beijing Chaoyang Hospital of Capital Medical University, and written informed consent with regard to the use of patient blood samples for research studies was obtained from all participants.

DNA extraction and genotyping. All participants provided $5 \mathrm{ml}$ venous blood, and blood samples were stored at $-20^{\circ} \mathrm{C}$ with $0.5 \mathrm{mg} / \mathrm{ml}$ EDTA as an anticoagulant, until required. Genomic DNA was extracted using the TIANamp Blood DNA kit (Tiangen Biotech Co., Ltd., Beijing, China), according to the manufacturer's instructions. Duplex polymerase chain reaction (PCR) with confronting two-pair primers was used for PCR-restriction fragment length polymorphism analysis in order to analyze the miR-146aG $>C$, miR-149C $>$ T, miR-196a2C $>$ T and miR-499A $>$ G genotypes The primers used and the products generated for the amplification of miR-146aG $>C$, miR-149C $>$ T, miR-196a $2 C>T$ and miR-499A $>$ G are shown in Table I.

The following PCR cycling conditions were used: An initial melting step of $5 \mathrm{~min}$ at $95^{\circ} \mathrm{C}$, followed by 35 cycles of denaturation at $94^{\circ} \mathrm{C}$ for $30 \mathrm{sec}$ and annealing at $64^{\circ} \mathrm{C}$ for $30 \mathrm{sec}$, with a final extension at $72^{\circ} \mathrm{C}$ for $10 \mathrm{~min}$. Reproducibility was verified using repeat analysis of a randomly selected subgroup of $10 \%$ of the subjects.
Statistical analysis. Data analysis was performed using SPSS version 10.0 (SPSS, Inc., Chicago, IL, USA) for Windows. Continuous variables are presented as the mean \pm standard deviation, and categorical variables are presented as frequencies and percentages. Differences in the distribution of demographic characteristics between the case and control groups were assessed using $\chi^{2}$ tests for the categorical data and Student's t-tests for the continuous variables. The $\chi^{2}$ test was used to compare the Hardy-Weinberg equilibrium of the genotype frequencies of $\mathrm{miR}-146 \mathrm{aG}>\mathrm{C}$, miR-149C $>\mathrm{T}$, $\mathrm{miR}-196 \mathrm{a} 2 \mathrm{C}>\mathrm{T}$ and $\mathrm{miR}-499 \mathrm{~A}>\mathrm{G}$ in the control group. The association between miR-146aG $>C$, miR-149C $>$, miR-196a2C $>$ T or miR-499A $>$ G polymorphisms and the risk of HCC was estimated using odds ratios (ORs) and their 95\% confidence intervals (CIs) from conditional logistic regression analyses. A homozygous genotype was used as the reference for calculating ORs. All P-values were two sided and $\mathrm{P}<0.05$ was considered to indicate a statistically significant difference.

\section{Results}

Among the 302 patients with HCC who were screened, 271 were included in the present study, with a participation rate of $89.7 \%$. For the control group, 568 individuals were screened and 532 were recruited into the present study, with a participation rate of $93.7 \%$. The HCC group consisted of 72 females and 199 males, while the control group consisted of 206 females and 326 males (Table II). The mean ages in the

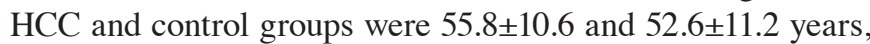
respectively. The patients with $\mathrm{HCC}$ were more likely to be male, with a higher age and incidence of $\mathrm{HBV}$ and $\mathrm{HCV}$ infection and a high probability of having a family history of cancer (All $\mathrm{P}<0.05$ ). No significant difference was observed in the smoking and drinking statuses of the individuals in the case group compared with those in the control group (All $\mathrm{P}>0.05)$. For the clinical characteristics, 204 patients $(75.3 \%)$ displayed liver cirrhosis, 151 (55.7\%) were TNM stage III-IV, $141(52.0 \%)$ were classified as Child-Pugh class C, 91 (33.6\%) 
Table II. Clinicopathological characteristics in the patients $(\mathrm{n}=271)$ with HCC and the control participants $(\mathrm{n}=532)$.

\begin{tabular}{|c|c|c|c|c|c|c|}
\hline Variables & Cases & $\%$ & Controls & $\%$ & $\chi^{2}$ or t-value & P-value \\
\hline Age, years $($ mean $\pm S D)$ & $55.8 \pm 10.6$ & - & $52.6 \pm 11.2$ & - & 3.89 & $<0.001$ \\
\hline \multicolumn{7}{|l|}{ Gender, $\mathrm{n}$} \\
\hline Male & 199 & 73.4 & 326 & 61.3 & - & - \\
\hline Female & 72 & 26.6 & 206 & 38.7 & 11.72 & 0.001 \\
\hline \multicolumn{7}{|l|}{ Smoking, $\mathrm{n}$} \\
\hline No & 173 & 63.8 & 354 & 66.5 & - & - \\
\hline Yes & 98 & 36.2 & 178 & 33.5 & 0.70 & 0.41 \\
\hline \multicolumn{7}{|l|}{ Drinking, $\mathrm{n}$} \\
\hline No & 182 & 67.2 & 389 & 73.1 & - & - \\
\hline Yes & 89 & 32.8 & 143 & 26.9 & 2.07 & 0.15 \\
\hline \multicolumn{7}{|l|}{ Family history of cancer, $\mathrm{n}$} \\
\hline No & 249 & 91.9 & 529 & 99.4 & - & - \\
\hline Yes & 22 & 8.1 & 3 & 0.6 & 37.04 & $<0.001$ \\
\hline \multicolumn{7}{|l|}{ Viral infection, $\mathrm{n}$} \\
\hline Both negative & 58 & 21.4 & 481 & 90.4 & - & - \\
\hline HBsAg-positive & 159 & 58.7 & 43 & 8.1 & - & - \\
\hline Anti-HCV Ab-positive & 49 & 18.1 & 8 & 1.5 & - & - \\
\hline Both positive & 5 & 1.8 & 0 & 0.0 & 389.37 & $<0.001$ \\
\hline \multicolumn{7}{|l|}{ Liver cirrhosis, $\mathrm{n}$} \\
\hline Absent & 204 & 75.3 & - & - & - & - \\
\hline Present & 67 & 24.7 & - & - & - & - \\
\hline \multicolumn{7}{|l|}{ TNM stage, $\mathrm{n}$} \\
\hline I-II & 151 & 55.7 & - & - & - & - \\
\hline III-IV & 120 & 44.3 & - & - & - & - \\
\hline \multicolumn{7}{|l|}{ Child-Pugh classification, $\mathrm{n}$} \\
\hline A & 39 & 14.4 & - & - & - & - \\
\hline B & 91 & 33.6 & - & - & - & - \\
\hline $\mathrm{C}$ & 141 & 52.0 & - & - & - & - \\
\hline \multicolumn{7}{|l|}{$\alpha$-Fetoprotein, $\mathrm{ng} / \mathrm{ml}$} \\
\hline$<100$ & 116 & 42.8 & - & - & - & - \\
\hline $100-400$ & 48 & 17.7 & - & - & - & - \\
\hline$>400$ & 107 & 39.5 & - & - & - & - \\
\hline
\end{tabular}

HCC, hepatocellular carcinoma; SD, standard deviation; HBsAg, hepatitis B surface antigen; Ab, antibody; HCV, hepatitis C virus; TNM, tumor-node-metastasis.

were classified as Child-Pugh class B, $116(42.8 \%)$ had $\alpha$-Fetoprotein levels of $<100 \mathrm{ng} / \mathrm{ml}$ and 107 (39.5\%) had levels of $>400 \mathrm{ng} / \mathrm{ml}$.

The allele and genotype distributions of miR-146aG $>C$, miR-149C>T, miR-196a2C > T and miR-499A > G were found to be in Hardy-Weinberg equilibrium in the control group (Table III). The miR-196a2C $>\mathrm{T}$ genotype frequency was significantly different in the individuals in the case group compared with those in the control group $\left(\chi^{2}=6.88 ; \mathrm{P}=0.032\right)$, while the frequencies of miR-146aG $>C$, miR-149C $>$ T and miR-499A $>$ G showed no significant differences between the case and control groups. Multivariate regression analyses revealed that subjects carrying the miR-196a2 TT genotype had a significantly reduced risk of HCC, with an adjusted
OR $(95 \% \mathrm{CI})$ of $0.62(0.38-0.99)$, and a significantly reduced risk was found in the dominant (OR, 0.69; 95\% CI, 0.49-0.98) and recessive (OR, 0.70; 95\% CI, 0.46-1.02) models. However, no significant association was found between the miR-146aG $>$ C, miR-149C $>$ T or miR-499A $>$ G polymorphisms and the risk of HCC.

Further analysis was performed on the interaction between miR-196a2C>T and the HBV and HCV infections (Table IV). Compared with the miR-196a2 CC genotype, individuals with HBV carrying the miR-196a2 CT and TT genotypes had a significantly reduced risk of $\mathrm{HCC}$, with an adjusted OR (95\% CI) of $0.62(0.41-0.95)$ and 0.39 (0.20-0.73), respectively. Moreover, the miR-196a $2 \mathrm{~T}$ allele was found to significantly reduce the risk of HCC by 0.33 -fold, compared 


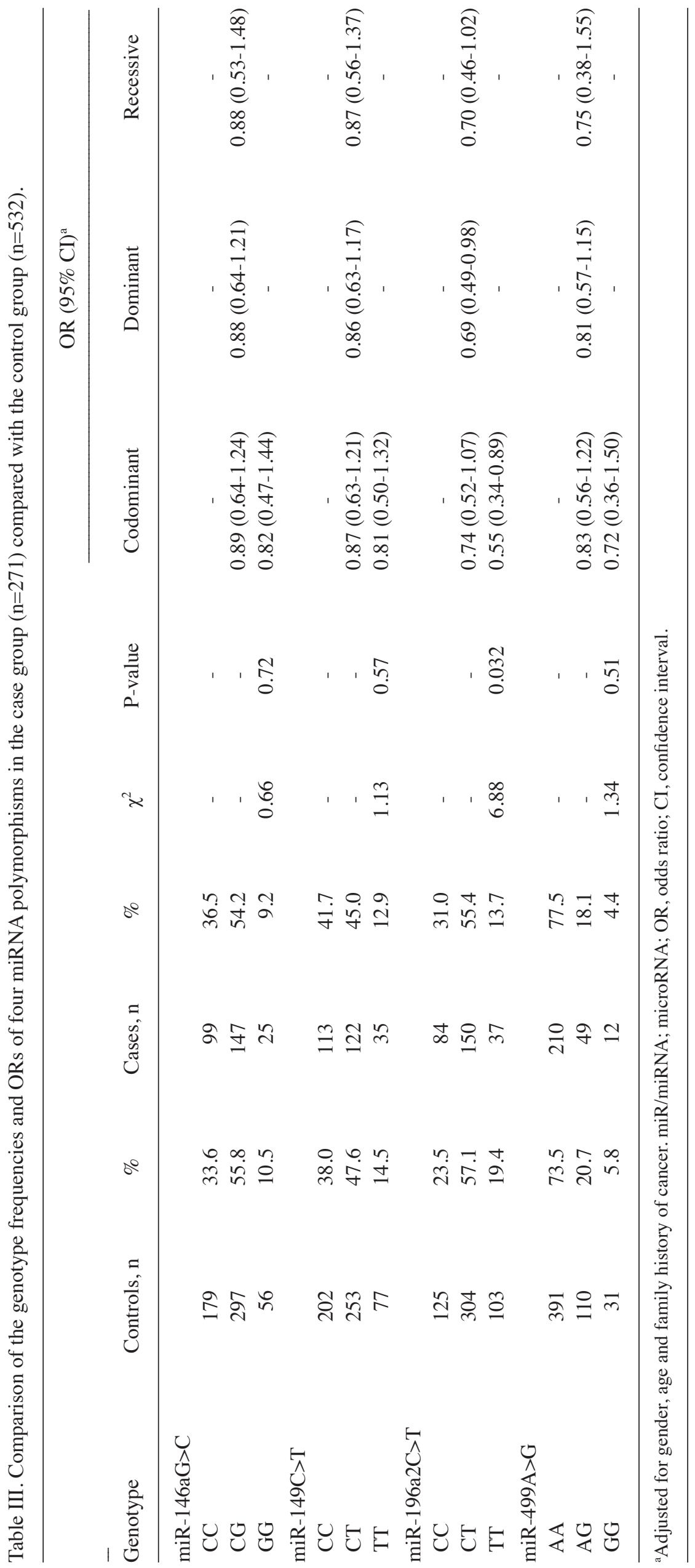


with the $\mathrm{C}$ allele. However, no significant association was found between miR-196a2C $>\mathrm{T}$ and $\mathrm{HCC}$ risk in patients with the HCV infection or those without HBV and HCV infection.

\section{Discussion}

In the present case-control study, the miR-196a2C $>\mathrm{T}$ polymorphism was found to be associated with a significantly reduced risk of HCC, however, miR-146aG $>C$, miR-149C $>\mathrm{T}$ and miR-499A $>\mathrm{G}$ were not associated with this risk. Furthermore, the miR-196a2C $>\mathrm{T}$ polymorphism was observed to significantly reduce the risk of $\mathrm{HCC}$ in patients infected with HBV.

It is well known that cancer is caused by multiple factors, including environmental and genetic factors. It has also been reported that miRNAs are a group of small non-coding RNA molecules, which have an important role in the development of cancer through regulating the expression of tumor suppressor genes (22). Previous clinical experimental studies have shown that polymorphisms in miR-122, $-196,-423$ and -499 are associated with the risk of hepatocellular carcinoma (23-25). The reasons for this may be that single nucleotide polymorphism (SNP) miRNA sequences can change the expression and maturation of miRNA, and thus, increase the risk of carcinogenesis (26).

The present study demonstrated that miR-196a2C $>\mathrm{T}$ was associated with HCC risk. It is reported that miR 196a2C $>\mathrm{T}$ polymorphisms have a role in the development of several types of cancer, including colorectal, breast, pancreastic, gastrointestinal and lung cancer (27-32). For HCC, only three studies have investigated the association between $\mathrm{miR} 196 \mathrm{a} 2 \mathrm{C}>\mathrm{T}$ polymorphisms and the development of $\operatorname{HCC}(15,16,33)$. In a study performed in Chinese, HBV-related patients with HCC showed that the risk of HCC was significantly higher in patients with the miR 196a2 CT genotype or T allele compared with those with the CC genotype (15). Furthermore, a study on 310 patients with $\mathrm{HCC}$ and 222 controls reported that the miR 196a2 CC genotype was associated with significantly increased mature miR 196a expression and that the miR 196a2 polymorphism may contribute to cirrhosis-related HCC susceptibility through affecting mature miR 196a expression (16). The present study found that the miR 196a 2 TT genotype and the $\mathrm{T}$ allele significantly reduced the risk of HCC compared with the CC genotype, which is in accordance with certain other previous studies $(15,16)$. However, in another study conducted in Chinese, HCC patients showed that the miR 196a2 TT genotype and the T allele greatly increased the risk of HCC (33). Furthermore, a recent study has shown that the miR 196a2C $>\mathrm{T}$ polymorphism may not be a HCC susceptibility factor, but may affect the effects of the HBV mutations (34). Discrepancies of these results may be due to differences in variant frequencies among various ethnic groups, and miR $196 \mathrm{a} 2 \mathrm{C}>\mathrm{T}$ polymorphisms may have different roles in the development of HCC depending on the population.

In the present study, miR-196a2C $>\mathrm{T}$ was found to interact with $\mathrm{HBV}$ infection. A previous study has shown that the miR-196a2C $>\mathrm{T}$ polymorphism is strongly affected by HBV mutations (34), which suggested that the HBV mutation may act synergistically with the miR-196a2C $>\mathrm{T}$ polymorphism.

The present study was performed in a single hospital and the number of patients included was relatively small. 
The relatively small sample size may decrease the statistical power of the investigation on the role of the miR-146aG $>C$, miRNA-149C $>$ T and miR-499A $>$ G genetic alterations. In the present study, a number if the patients who were newly diagnosed with HCC left the study. This included patients who moved to other hospitals or did not agree to provide genetic information and blood samples. Thus, further large, multicenter investigations are required to confirm the association between SNPs in miRNA and the risk of HCC.

In conclusion, in the present study, the miR-196a2C>T polymorphism was found to have a protective role in patients with HCC, particularly in those with HBV infection. However, no association was found between the miR-146aG $>C$, miRNA-149C $>$ T or miR-499A $>$ G polymorphisms and the risk of HCC. SNPs in miRNA sequences may be used as diagnostic biomarkers for HCC. Further large-sample investigations are required to investigate the role of SNPs in miRNA sequences in the development of HCC.

\section{References}

1. International Agency for Research on Cancer (2012): Liver Cancer. Estimated Incidence, Mortality and Prevalence Worldwide in 2012. http://globocan.iarc.fr/Pages/fact_sheets_cancer.aspx. Accessed January 1, 2014.

2. Yu MC and Yuan JM: Environmental factors and risk for hepatocellular carcinoma. Gastroenterology 127 (5 Suppl 1): S72-S78, 2004.

3. Davila JA, Morgan RO, Shaib Y, McGlynn KA and El-Serag HB: Hepatitis Cinfection and the increasingincidence of hepatocellular carcinoma: a population-based study. Gastroenterology 127: 1372-1380, 2004.

4. Bartel DP: MicroRNAs: genomics, biogenesis, mechanism, and function. Cell 116: 281-297, 2004

5. Valencia-Sanchez MA, Liu J, Hannon GJ, et al: Control of translation and mRNA degradation by miRNAs and siRNAs. Genes Dev 20: 515-524, 2006.

6. Lim LP, Lau NC, Garrett-Engele P, et al: Microarray analysis shows that some microRNAs downregulate large numbers of target mRNAs. Nature 433: 769-773, 2005.

7. Wilfred BR, Wang WX and Nelson PT: Energizing miRNA research: a review of the role of miRNAs in lipid metabolism, with a prediction that miR-103/107 regulates human metabolic pathways. Mol Genet Metab 91: 209-217, 2007.

8. Pizzini S, Bisognin A, Mandruzzato S, et al: Impact of microRNAs on regulatory networks and pathways in human colorectal carcinogenesis and development of metastasis. BMC Genomics 14: 589, 2013.

9. Sung SY, Liao CH, Wu HP, et al: Loss of let-7 microRNA upregulates IL-6 in bone marrow-derived mesenchymal stem cells triggering a reactive stromal response to prostate cancer. PLoS One 8: e71637, 2013.

10. Saito K, Inagaki K, Kamimoto T, et al: MicroRNA-196a is a putative diagnostic biomarker and therapeutic target for laryngeal cancer. PLoS One 8: e71480, 2013.

11. He B, Pan Y, Cho WC, et al: The association between four genetic variants in microRNAs (rs11614913, rs2910164, rs3746444, rs2292832) and cancer risk: evidence from published studies. PLoS One 7: e49032, 2012.

12. Hu Z, Liang J, Wang Z, et al: Common genetic variants in pre-microRNAs were associated with increased risk of breast cancer in Chinese women. Hum Mutat 30: 79-84, 2009.

13. Lee HC, Kim JG, Chae YS, et al: Prognostic impact of microRNA-related gene polymorphisms on survival of patients with colorectal cancer. J Cancer Res Clin Oncol 136: 1073-1078, 2010.
14. Peng S, Kuang Z, Sheng C, et al: Association of microRNA-196a-2 gene polymorphism with gastric cancer risk in a Chinese population. Dig Dis Sci 55: 2288-2293, 2010.

15. Qi P, Dou TH, Geng L, Zhou FG, Gu X, Wang H and Gao CF: Association of a variant in MIR 196A2 with susceptibility to hepatocellular carcinoma in male Chinese patients with chronic hepatitis B virus infection. Hum Immunol 71: 621-626, 2010.

16. $\mathrm{Li} \mathrm{XD}$, Li ZG, Song $\mathrm{XX}$ and Liu CF: A variant in microRNA-196a2 is associated with susceptibility to hepatocellular carcinoma in Chinese patients with cirrhosis. Pathology 42: 669-673, 2010.

17. Xiang Y, Fan S, Cao J, et al: Association of the microRNA-499 variants with susceptibility to hepatocellular carcinoma in a Chinese population. Mol Biol Rep 39: 7019-7023, 2012.

18. Kim WH, Min KT, Jeon YJ, et al: Association study of microRNA polymorphisms with hepatocellular carcinoma in Korean population. Gene 504: 92-97, 2012.

19. Hu M, Zhao L, Hu S, Yang J: The association between two common polymorphisms in MicroRNAs and hepatocellular carcinoma risk in Asian population. PLoS One 8: e57012, 2013.

20. Zheng SQ, Li YX, Zhang Y, et al: MiR-101 regulates HSV-1 replication by targeting ATP5B. Antiviral Res 89: 219-226, 2011.

21. Cui C, Griffiths A, Li G, et al: Prediction and identification of herpes simplex virus 1-encoded microRNAs. J Virol 80: 5499-5508, 2006.

22. Esquela-Kerscher A and Slack FJ: Oncomirs - microRNAs with a role in cancer. Nat Rev Cancer 6: 259-269, 2006.

23. Spaniel C, Honda M, Selitsky SR, et al: microRNA-122 abundance in hepatocellular carcinoma and non-tumor liver tissue from Japanese patients with persistent HCV versus HBV infection. PLoS One 8: e76867, 2013.

24. Ma Y, Wang R, Zhang J, et al: Identification of miR-423 and miR-499 polymorphisms on affecting the risk of hepatocellular carcinoma in a large-scale population. Genet Test Mol Biomarkers: May 22, 2014.

25. Kim HY, Yoon JH, Lee HS, et al: MicroRNA-196A-2 polymorphisms and hepatocellular carcinoma in patients with chronic hepatitis B. J Med Virol 86: 446-453, 2014.

26. Wu M, Jolicoeur N, Li Z, et al: Genetic variations of microRNAs in human cancer and their effects on the expression of miRNAs. Carcinogenesis 29: 1710-1716, 2008.

27. Zhang M, Jin M, Yu Y, et al: Associations of miRNA polymorphisms and female physiological characteristics with breast cancer risk in Chinese population. Eur J Cancer Care (Engl) 21: 274-280, 2012.

28. Wang F, Sun GP, Zou YF, Fan LL and Song B: Quantitative assessment of the association between miR-196a2 rs11614913 polymorphism and gastrointestinal cancer risk. Mol Biol Rep 40: 109-116, 2013.

29. Hezova R, Kovarikova A, Bienertova-Vasku J, et al: Evaluation of SNPs in miR-196-a2, miR-27a and miR-146a as risk factors of colorectal cancer. World J Gastroenterol 18: 2827-2831, 2012.

30. Pavlakis E, Papaconstantinou I, Gazouli M, et al: MicroRNA gene polymorphisms in pancreatic cancer. Pancreatology 13: 273-278, 2013.

31. Wei J, Zheng L, Liu S, et al: MiR-196a2 rs11614913 T>C polymorphism and risk of esophageal cancer in a Chinese population. Hum Immunol 74: 1199-1205, 2013.

32. Yuan Z, Zeng X, Yang D, Wang W and Liu Z: Effects of common polymorphism rs11614913 in Hsa-miR-196a2 on lung cancer risk. PLoS One 8: e61047, 2013.

33. Hao YX, Wang JP and Zhao LF: Associations between three common MicroRNA polymorphisms and hepatocellular carcinoma risk in Chinese. Asian Pac J Cancer Prev 14: 6601-6604, 2013.

34. Han Y, Pu R, Han X, et al: Associations of pri-miR-34b/c and pre-miR-196a2 polymorphisms and their multiplicative interactions with hepatitis B virus mutations with hepatocellular carcinoma risk. PLoS One 8: e58564, 2013. 